\title{
La desagregación tecnológica como herramienta para la generación de políticas públicas de incentivo a la innovación
}

\author{
Héctor Sebastián Naranjo Silva \\ Universidad Tecnológica Indoamérica (Quito-Ecuador) \\ sebastianaranjo@hotmail.com \\ Juan Carlos Salvador Jiménez \\ Universidad Internacional SEK (Quito-Ecuador) \\ juankrloss@hotmail.com
}

Fecha de presentación: 13 diciembre de 2016 • Fecha de aceptación: 25 de febrero de 2017 Artículo de investigación 


\section{Resumen}

El artículo analiza el potencial que la desagregación tecnológica tiene para la generación de políticas públicas que incentiven la innovación, como una herramienta técnica para la asimilación de la tecnología. Se consideran mecanismos que los países de la región usan para fomentar la innovación y que permitan el desarrollo de productos con el fin de pasar de una economía extractivista a una enfocada en servicios innovadores. El principal problema encontrado a nivel regional es la falta de normativa y exceso de burocracia que no promueven ambientes innovadores y tecnológicos.

Palabras clave: Desagregación, innovación, tecnología, política pública, paquete tecnológico. JEL: L5 Regulaciones y política industrial; O3 Cambio tecnológico.

\section{Abstract}

The article analyses the potential that the technological disaggregation has for the generation of public policies that encourage innovation, as a technical tool for the assimilation of technology. Mechanisms that the countries of the region use to promote innovation and that allow the development of products in order to move from an extractive economy to one focused on innovative services are considered. The main problem found at the regional level is the lack of regulations and excessive bureaucracy that do not promote innovative and technological environments.

Keywords: Disaggregation, innovation, technology, public policy, technological package.

JEL: L5 Regulations and Industrial Policy; O3 Technological change.

\section{Resumo}

$\mathrm{O}$ artigo analisa o potencial da desagregação tecnológica para a geração de políticas públicas que incentivem a inovação, como uma ferramenta técnica para a assimilação da tecnologia. Foram considerados os mecanismos que os países da região usam para fomentar a inovação e que permitem o desenvolvimento de produtos com a finalidade de se passar de uma economia extrativista a uma economia focada em serviços inovadores. O principal problema encontrado em um nível regional foi a falta de normativa e o excesso de burocracia que não promovem ambientes inovadores e tecnológicos.

Palavras-chave: Separação, inovação, tecnologia, políticas públicas, pacote de tecnologia. JEL: L5: Regulamentação e Política Industrial; O3 A mudança tecnológica. 


\section{Introducción}

D la desagregación tecnológica una herramienta funcional para la - generación de políticas públicas que beneficien el desarrollo industrial de un país? ¿Las grandes firmas comerciales permiten la liberación de tecnología como aporte para la sociedad? ¿Las patentes permiten un adecuado desarrollo industrial en un país? Estas preguntas que a simple vista parecen vanas o sin sentido merecen un análisis profundo, debido a que actualmente el desarrollo industrial es uno de los principales indicadores de bienestar de un país. El presente artículo establece un análisis sobre el impacto que genera la desagregación tecnológica en el desarrollo industrial de un país, así como los principales problemas y beneficios generados en diferentes países de la región, y busca poner en contexto los diferentes esfuerzos de los países para generar políticas públicas que permitan la asimilación tecnológica y el desarrollo innovador.

Independientemente de la vinculación laboral muchas personas han escuchado acerca de los términos desagregación tecnológica, tecnologías periféricas, tecnologías medulares o paquete tecnológico. El alcance de estos términos será definido a continuación con el objetivo de tener una visión clara sobre el impacto del tema, planteado en el presente artículo.

La desagregación tecnológica hace referencia, en grandes rasgos, al desarticular, desunir o abrir un elemento grande en pequeñas partes; desde una visión técnica intervienen las tecnologías medulares que por definición son todos los conocimientos necesarios e indispensables para que el objeto desarrolle sus características de forma correcta; las tecnologías periféricas, por otra parte, son los conocimientos no esenciales o imprescindibles para que el objeto cumpla con su función; al conjunto de documentos, derechos de propiedad, actividades, soluciones de gestión y modelos necesarios para lograr que una tecnología se transforme en un producto comercializable o transferible se denomina paquete tecnológico. 
La desagregación tecnológica es el "proceso dirigido a descomponer proyectos, en sus diferentes elementos técnicos y económicos con el objeto de permitir la apertura de varias licitaciones para su ejecución buscando la participación de la industria y el trabajo nacional" (EPI 2014, 4). Otros autores definen a la desagregación tecnológica como "un proceso usado para descomponer proyectos, estudiando las características técnicas del proyecto y de cada uno de los componentes, en relación a la capacidad tecnológica del sistema productivo del país, con el fin de mejorar la posición de negociación, aprovechar la oferta nacional y determinar la participación nacional" (Rivera y Zárate 2015, 38).

El conocimiento aprendido o know-how tecnológico que existe alrededor de un desarrollo innovativo -sea en un producto o proceso- puede ser aplicado con la desagregación tecnológica cuya viabilidad comercial y de mercado ha sido probada fehacientemente para rendir beneficios económicos a las partes interesadas, además de asimilar la tecnología por lo que se dirige a descomponer los proyectos en sus diferentes elementos técnicos y económicos, buscando la participación de la industria y el trabajo nacional.

La desagregación tecnológica está muy enlazada con la transformación de la tecnología debido a que es un proceso por el cual se identifican, categorizan y caracterizan las necesidades y demandas tecnológicas de los productores -en un sector determinado- y se formulan soluciones; además, es un mecanismo para lograr que la tecnología sea rápidamente incorporada al mercado de productos y servicios.

A grandes rasgos, la desagregación tecnológica es un instrumento de política industrial que busca maximizar la participación propia de un país mediante la ejecución de proyectos de diversa índole, por medio del incremento del componente tecnológico nacional en la producción de un bien o servicio.

Se sabe que las distancias en el conocimiento tecnológico que separan al mundo desarrollado del mundo en desarrollado son bastante amplias y esas distancias no cesan de crecer. Cada vez se acentúa más el monopolio científico y tecnológico de los países industrializados que guardan celosamente los secretos de su conocimiento a través de rigurosos sistemas de marcas y patentes, pero dentro de la competitividad y de un mundo consumista el mercado demanda más calidad. 
Los países en vía de desarrollo han adoptado diferentes políticas de transferencia de tecnología para protegerse del monopolio tecnológico; dentro de ellas, la desagregación tecnológica es un mecanismo que busca abrir los paquetes cerrados en que viene la tecnología extranjera y disociar sus diferentes partes de manera que los profesionales y expertos locales trabajen en aquellas, fomentando la investigación nacional, abriendo espacio para la práctica de los técnicos locales, lo que contribuye a generar en los países receptores la capacidad de seleccionar, adaptar y perfeccionar la tecnología importada.

La esencia de la desagregación tecnológica es descomponer el paquete tecnológico que se ofrece desde afuera, de modo de que el país receptor importe solamente los elementos que le hacen falta y aporte localmente los que conoce; de esta manera "se pretende impulsar la participación de empresas o industrias nacionales, favoreciendo el aprendizaje, la investigación, innovación y autonomía" (Flores 2008, 7).

Las metodologías desarrolladas sobre la forma de generar la desagregación tecnológica son varias. El presente artículo muestra un modelo basado en el marco legal internacional.

Históricamente, la desagregación tecnológica ha sido realizada mediante el método de desagregación estructural de productos (PBS por sus siglas en inglés) (Haugan 2012), desarrolladada en Estado Unidos, y aplicable aspectos como productos, servicios y elementos administrativos. La metodología descompone en subconjuntos, sistemas, componentes tecnológicos, partes y elementos un bien o servicio para incorporar la diagramación por jerarquización y multinivel que propone la PBS, para definir los diferentes subconjuntos, partes y elementos que conforman un objeto y propone la manera mas conveniente para realizar categorizaciones como: listado de materiales, componentes del sistema y subsistemas.

La metodología PBS muestra facilidad de uso y orden lógico de procedimientos, considera de manera jerárquica los componentes que conforman un producto en general independiente de su naturaleza y la industria a la que pertenece. Además, es una herramienta que ayuda a la reducción de complejidad de todo proceso, facilita la comprensión y definición de cada uno de los componentes de manera ordenada, por lo que es de utilidad para la planificación, coordinación y control en la desagregación tecnológica. 
La metodología fue adoptada en Ecuador por el Ministerio de Industrias y Productividad y permite generar una base de datos con el propósito de definir políticas públicas que favorezcan la producción y desarrollo tecnológico ecuatoriano en aras del cambio de la matriz productiva.

La metodología de desagregación tecnológica utilizada en Ecuador pretende generar información sobre qué bienes y servicios son más adquiridos a nivel nacional, enfocándose de manera puntual en las compras periódicas realizadas por el Estado. El conocimiento del mercado permite generar encadenamientos productivos entre empresarios locales y con la implementación de esta metodología se prevé contar con información adicional de manufactura y tipos de ensamble existentes en la industria nacional, para posteriormente poder aplicar, en orden secuencial, los siguientes pasos:

1. Desagregación descriptiva: implica el reconocimiento de los procesos de manufactura de todos y cada uno de los componentes de un equipo objeto de estudio.

2. Desagregación cualitativa: toma en cuenta los lineamientos a los que se rige el producto como normas, certificaciones y proveedores nacionales que tienen capacidad de suministrar componentes.

3. Desagregación cuantitativa: busca determinar los costos de producción, mano de obra y servicios para la fabricación de los componentes y partes del paquete tecnológico que desagregan.

4. Desagregación por ingeniería inversa: del conjunto, como todo de un bien, se reduce al mínimo posible de partes que lo integran; dentro de este paso se considera la ingeniería de elementos, partes y componentes en el desarrollo de planos de detalle de ingeniería para generar prototipos con componente nacional.

Además del análisis conceptual de bienes y servicios con las metodologías de desagregación descritas, es factible utilizar diversos paquetes informáticos que facilitarían la desagregación descriptiva, los que permiten generar, de manera ordenada, esquemas y diagramas, para unificar criterios con el fin de promover políticas públicas beneficiosas para el sector industrial.

A continuación se detallan las características más relevantes de la metodología PBS y se identifican los requerimientos mínimos para realizar un análisis de desagregación tecnológica. 


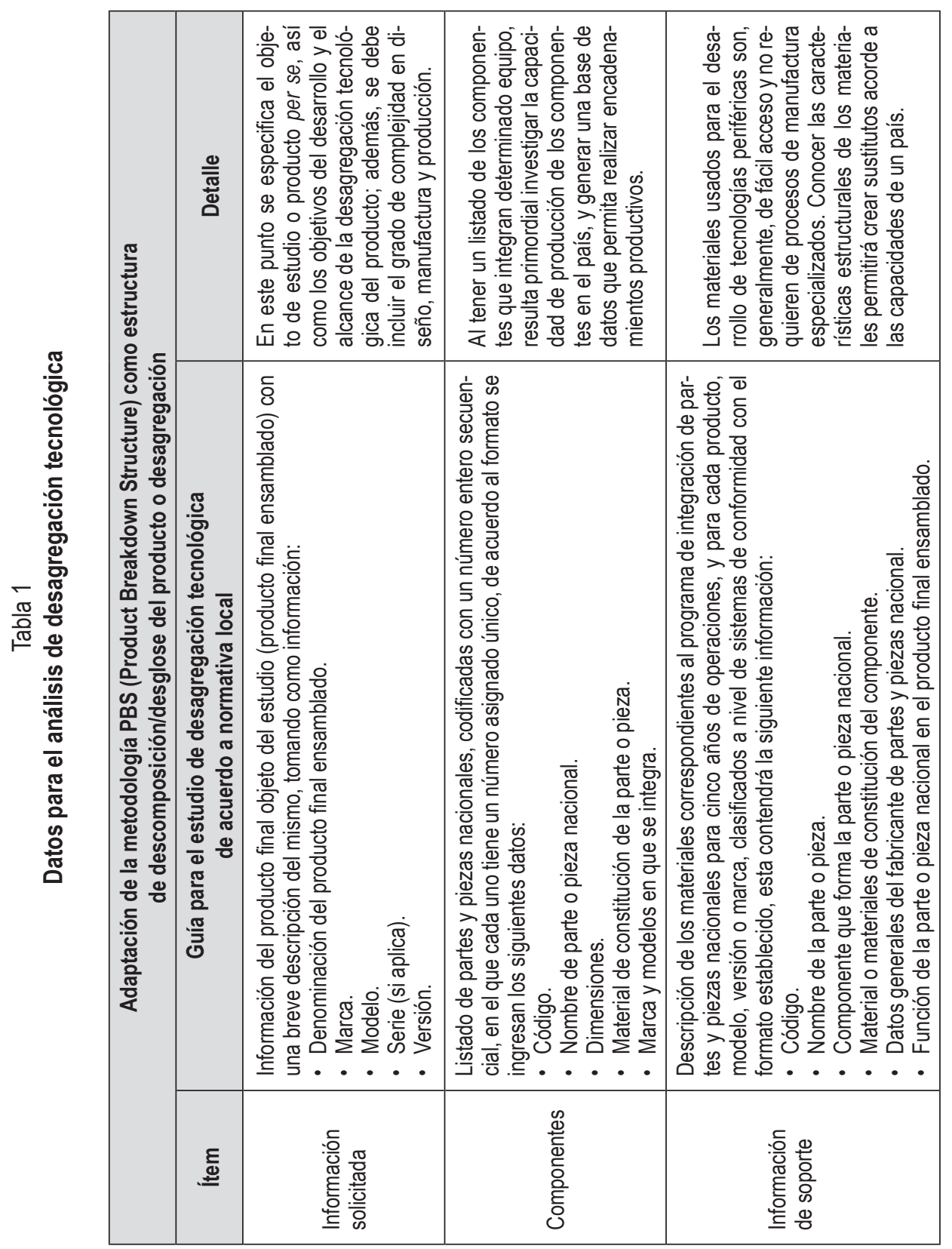




\begin{tabular}{|c|c|}
\hline 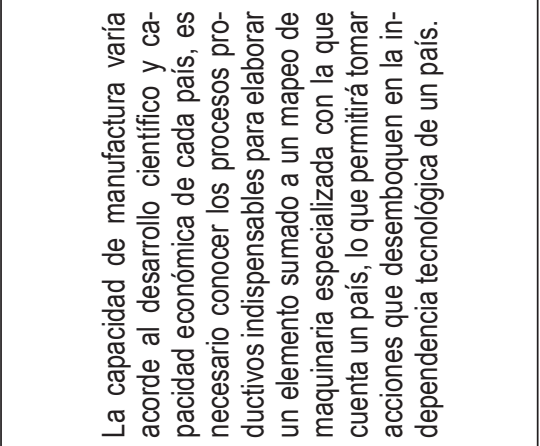 & 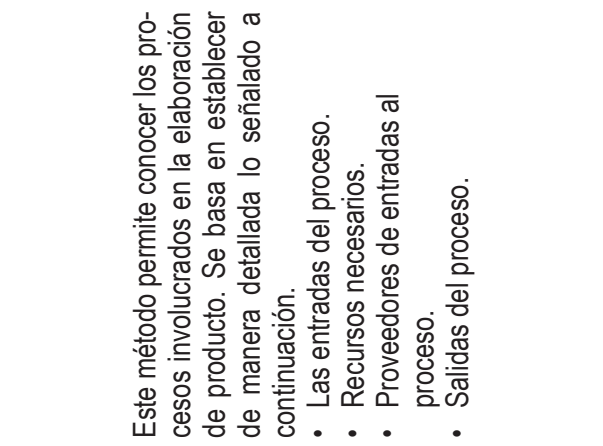 \\
\hline 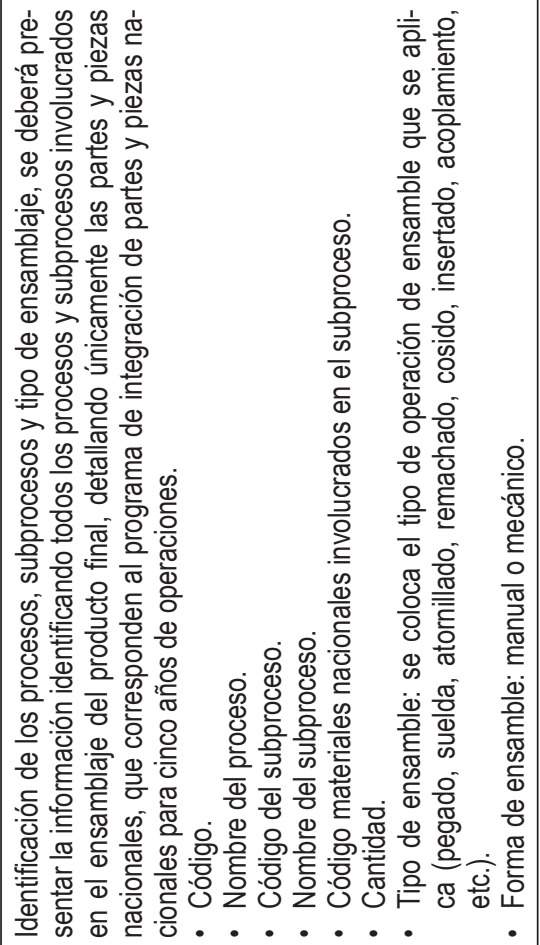 & 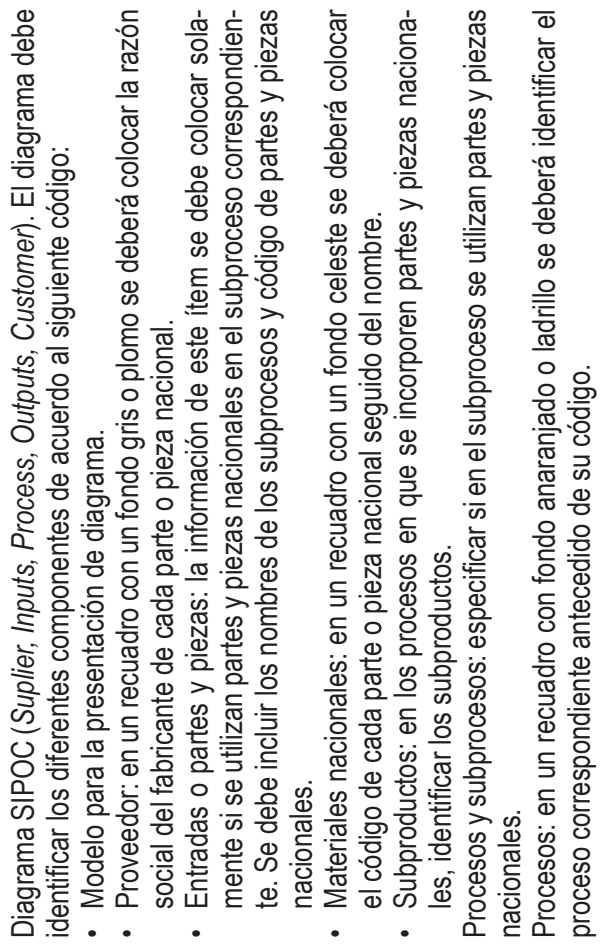 \\
\hline 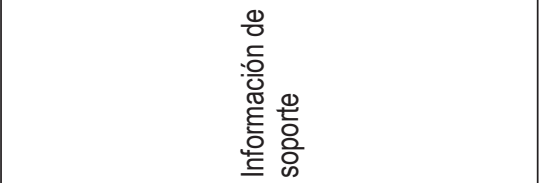 & 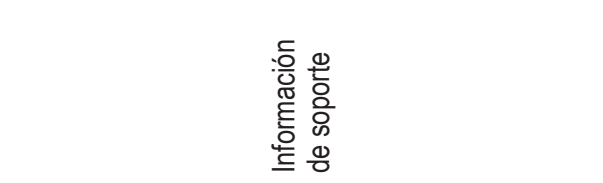 \\
\hline
\end{tabular}




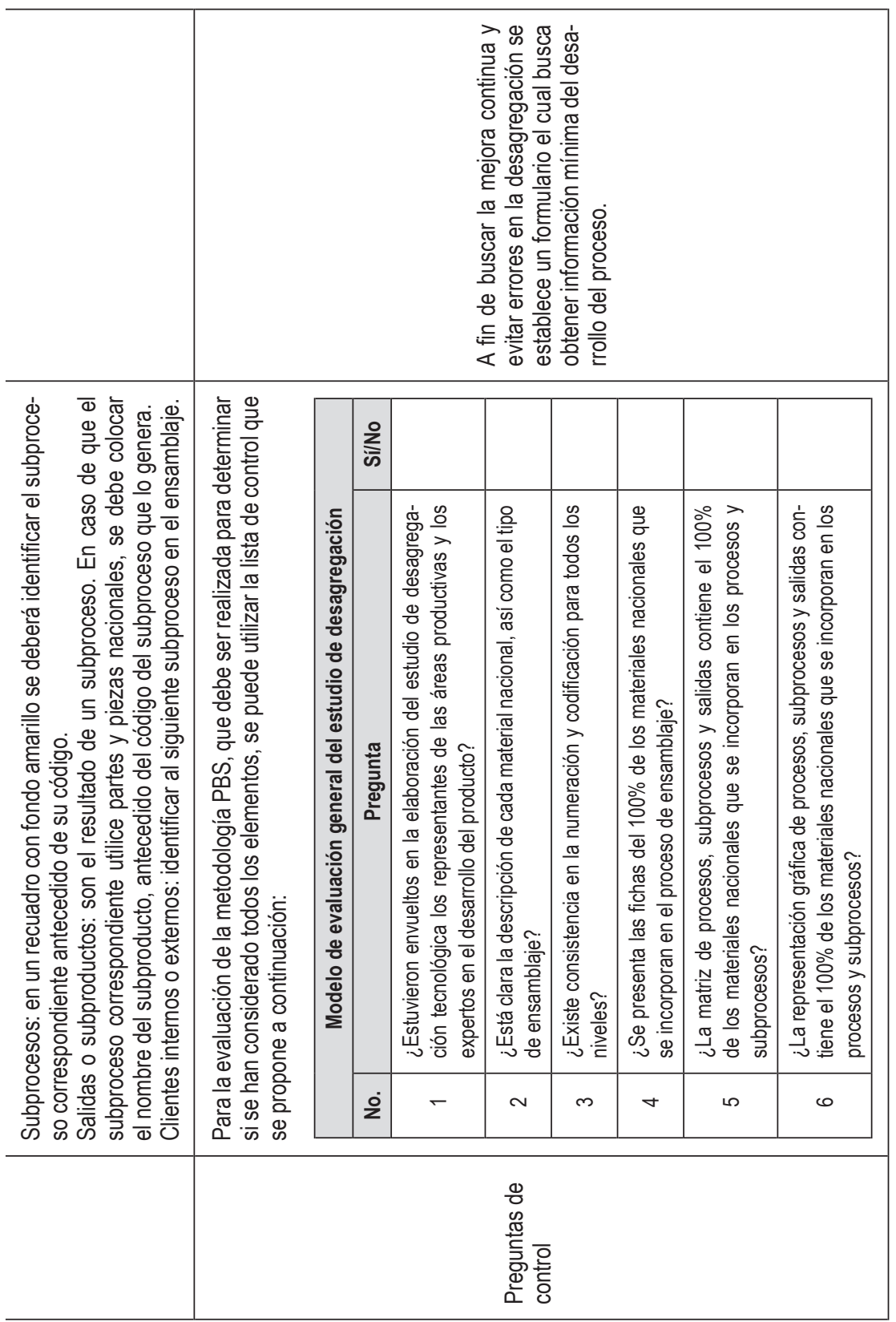




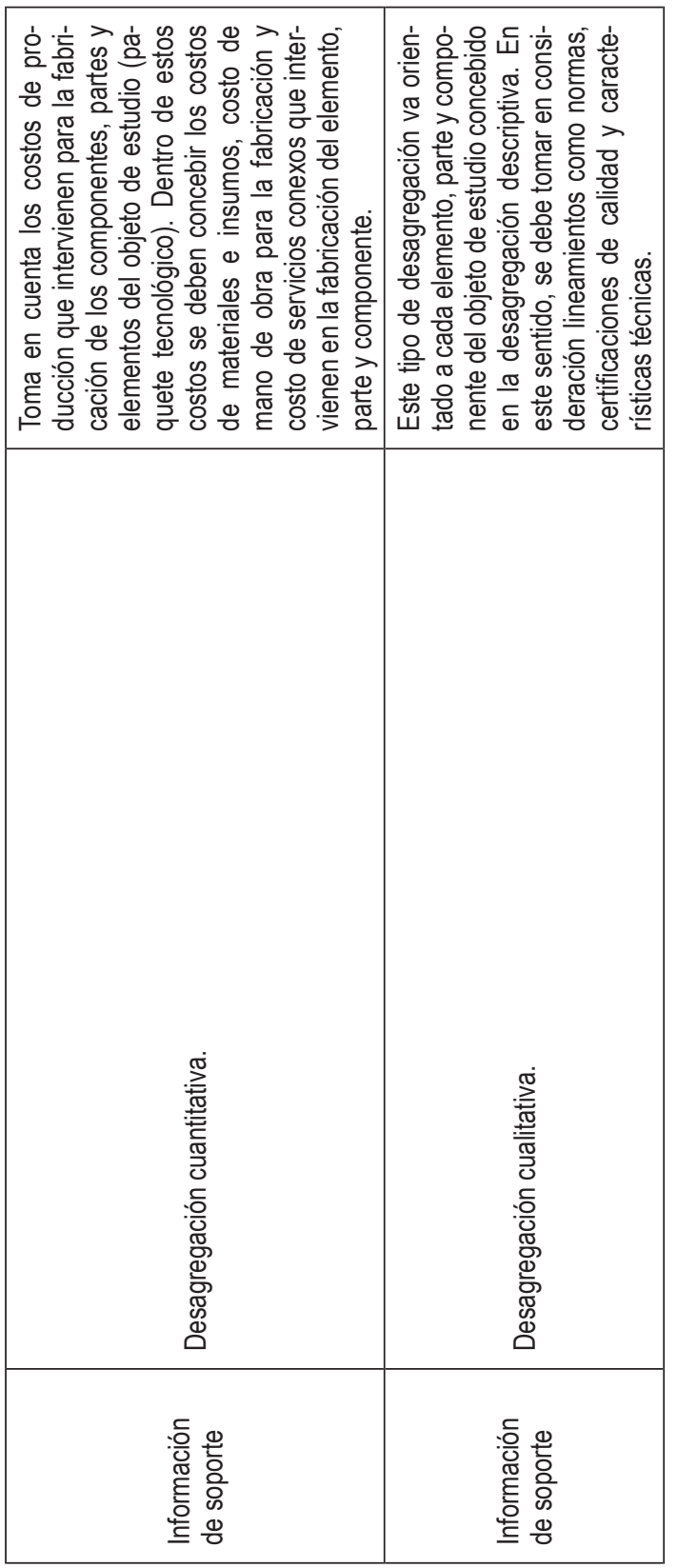

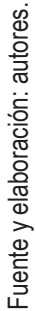


La tabla 1 explica, en una secuencia de pasos, los requisitos, datos e información necesaria para elaborar un análisis completo de desagregación tecnológica. Realizar un proceso de desagregación de manera secuencial, como lo indicado en la tabla 1, permite un mejor aprovechamiento de recursos que beneficia la administración del proyecto.

Para una mejor concepción del proceso de desagregación es necesario conocer de primera mano los procesos constructivos utilizados así como los equipos y materiales que conforman el ensamblaje.

Finalmente, se recomienda recurrir a métodos de ingeniería inversa y técnicas no destructivas para conservar la integridad y funcionalidad del equipo, por lo tanto el análisis dimensional y de tolerancia es limitado.

\section{Análisis del marco legal nacional e internacional}

\section{Contexto ecuatoriano}

Las políticas públicas ecuatorianas sobre temas de desagregación tecnológica son varias, desde la reforma a la Constitución de la República en el año 2008, la cual establece acciones que buscan sustituir las importaciones por un modelo de industrialización, como el Plan Nacional del Buen Vivir que en su objetivo ocho busca consolidar el sistema económico social y solidario de forma sostenible (SENPLADES 2013), es decir, habla sobre el desarrollo de productos y bienes locales para sustituir las importaciones paulatinamente, generando institucionalidad en el país, priorizando los productos nacionales, estableciendo requisitos de desempeño a la industria para generar encadenamientos, generando puestos de trabajo y promoviendo el uso de la desagregación y transferencia tecnológica así como la reinversión de utilidades.

Otro sustento legal es el cambio de la matriz productiva mediante el impulso de los sectores estratégicos; esta política busca la oferta de bienes y servicios que se oriente hacia la diversificación productiva basada en la incorporación de valor agregado nacional, impulso de las exportaciones y su expansión en productos y destinos incluyendo actores en la desconcentración de la producción. 
Ecuador ha creado varias iniciativas a favor de la asimilación de tecnología; según el numeral 10 del artículo 6 de la Ley Orgánica del Sistema Nacional de Contratación Pública (LOSNCP), la definición formal de la desagregación tecnológica expresa que

Es el estudio pormenorizado que realiza la Entidad Contratante en la fase pre contractual, en base a la normativa y metodología definida por el Servicio Nacional de Contratación Pública en coordinación con el Ministerio de Industrias y Productividad, sobre las características técnicas del proyecto y de cada uno de los componentes objeto de la contratación, en relación a la capacidad tecnológica del sistema productivo del país, con el fin de mejorar la posición de negociación de la Entidad Contratante, aprovechar la oferta nacional de bienes, obras y servicios acorde con los requerimientos técnicos demandados, y determinar la participación nacional" (Losncp 2013, 5).

Actualmente el MIPRO, mediante la implementación de un centro de desagregación tecnológica, busca impulsar la investigación y desarrollo tecnológico en beneficio de la industria usando la desagregación tecnológica e ingeniería inversa, mecanismos enfocados en elevar la matriz productiva con apoyo a la empresa local, al mismo tiempo que se busca fomentar estrategias de producción nacional de partes y piezas además de desarrollar prototipos de componentes, de paquetes tecnológicos de bienes, obras y servicios.

\section{Acuerdo de Cartagena}

En el año de 1974, con el Acuerdo de Cartagena, se establecieron diferentes procedimientos con la finalidad de lograr la independencia tecnológica de los países de la región, la Decisión 84 establece temas como la desagregación tecnológica e impulsa a los países miembros a la utilización de recursos humanos, financieros, científicos, técnicos y de infraestructura para generar acciones concretas en cuanto a la programación de actividades científicas y tecnológicas así como la copia, asimilación y adaptación de tecnologías extranjeras.

En cumplimiento de lo expuesto, varios países de la región han establecido mecanismos que permitan la asimilación de tecnología, entre las principales actividades realizadas esta la creación de normativa legal y la creación de centros de desagregación tecnológica. 


\section{Desagregación en la región}

Para complementar, la desagregación en países vecinos también ha avanzado, por ejemplo, en 1991, mediante Decreto Presidencial 0591 (Cadavid, Martínez y Monsalve 2002), Colombia busca establecer un reglamento que permita una asimilación coordinada de la tecnología extranjera, buscando pactar medidas conducentes para los efectos de la transferencia tecnológica. El Departamento Administrativo de Ciencia, Tecnología e Innovación es la institución encargada de fomentar el desarrollo científico, tecnológico y para la innovación como ejes fundamentales del desarrollo social y productivo del país.

En el 2001, en Venezuela, se promulgó la "Ley de Ciencia, Tecnología e Innovación de Venezuela" (Chávez e Innovación 2001), que busca desarrollar los principios orientadores en materia de ciencia, tecnología e innovación así como la implantación de mecanismos institucionales y operativos para la promoción, estímulo y fomento de la investigación científica, la apropiación social del conocimiento y la transferencia e innovación tecnológica a fin de fomentar la capacidad para la generación, uso y circulación del conocimiento e impulsar el desarrollo.

En diciembre de 2007, Perú expidió la "Ley Marco de Ciencia, Tecnología e Innovación Tecnológica", que pretende normar el desarrollo, promoción, consolidación, difusión y transferencia de la tecnología en el país. Además el Consejo Nacional de Ciencia, Tecnología e Innovación Tecnológica es la institución "rectora del Sistema Nacional de Ciencia y Tecnología e Innovación Tecnológica, SINACYT, integrada por la Academia, los Institutos de Investigación del Estado, las organizaciones empresariales, las comunidades y la sociedad civil" (Perú 2007, 4).

Finalmente, Bolivia cuenta con el Viceministerio de Ciencia y Tecnología, que es la institución encargada de desarrollar una cultura del conocimiento basada en el acceso, intercambio y generación de información científica y tecnológica (Cadavid, Martínez y Monsalve 2002).

\section{Fomento de la innovación}

Debido a que existen diferentes factores que limitan el desarrollo de innovación, en los países de la región se torna necesario que los gobiernos asu- 
man la responsabilidad directa en el tratamiento de estos y deberían indagar sobre la percepción que existe alrededor de la innovación en las instituciones estatales involucradas en temas de fomento a la industria para proponer alternativas de solución en caso de encontrar desatinos.

Conocer fortalezas y debilidades del talento humano, en las instituciones del Estado involucradas -en temas relacionados a innovación-, permite tomar acciones para fortificar el personal y por ende mejorar la prestación de su servicio, además de realizar un trabajo óptimo con el cliente externo que requiere asesoría o acompañamiento en el desarrollo de proyectos innovadores.

Existen varios estudios que analizan los factores que limitan la innovación, para un análisis de percepción se propone utilizar los factores más relevantes:

\section{Tabla 2}

Factores que limita e impulsan la innovación

\begin{tabular}{|c|c|}
\hline $\begin{array}{l}\text { Factores que limitan } \\
\text { el desarrollo de la innovación }\end{array}$ & $\begin{array}{l}\text { Factores que impulsan el desarrollo } \\
\text { de la innovación }\end{array}$ \\
\hline $\begin{array}{l}\text { - Denominación del producto final } \\
\text { ensamblado. } \\
\text { - Baja disponibilidad en el suministro } \\
\text { de materias primas. } \\
\text { - Productos importados } \\
\text { de baja calidad y precio } \\
\text { (competencia desleal). } \\
\text { - Informalidad y escasa visibilidad. } \\
\text { - Poco o nulo conocimiento del } \\
\text { - } \text { Altos costos de producción. } \\
\text { - Falta de mano de obra calificada. } \\
\text { - Falta de laboratorios acreditados. } \\
\text { - Desconocimiento en gestión de } \\
\text { - Lspectos ambientales. } \\
\text { - Limitaciones en gestión y vigilancia } \\
\text { tecnológica. } \\
\text { Falta de canales de comercialización. }\end{array}$ & $\begin{array}{l}\text { - Apoyo a la investigación, innovación } \\
\text { y transferencia de conocimiento y tecnología } \\
\text { aplicada a las necesidades productivas. } \\
\text { - Generación de condiciones que promuevan la } \\
\text { diversificación productiva y la generación de valor } \\
\text { agregado. } \\
\text { - Incentivo a la demanda de bienes nacionales. } \\
\text { - Impulso a la generación de estrategias } \\
\text { que permitan el acceso al mercado nacional } \\
\text { - e internacional. } \\
\text { - Generación de condiciones favorables para el } \\
\text { emprendimiento. } \\
\text { - Apoyo a la inversión productiva nacional. } \\
\text { - } \text { Fespaldo a grupos asociativos y comunitarios. } \\
\text { y economías locales. } \\
\text { - Apoyo a la creación de infraestructura } \\
\text { de almacenamiento, acopio, vialidad, } \\
\text { accesibilidad, conectividad, logística, movilidad. }\end{array}$ \\
\hline
\end{tabular}

Fuente y elaboración: autores. 
El fomento de centros de desagregación tecnológica e innovación industrial, en los países en vías de desarrollo, adecuadamente equipados y administrados, permitirá un impulso cualitativo en relación con la asimilación de tecnología, evitar la fuga de talentos con incentivos económicos y técnicos permitirá un desarrollo armónico de proyectos de investigación; medidas estatales a favor de estos mecanismos permitirán un crecimiento científico y tecnológico de la región.

\section{Para cerrar: problemas encontrados}

Los esfuerzos de los distintos países están enfocados en la creación de normativa y acciones burocráticas que poco influyen en el desarrollo de tecnología, en la región se mantiene una especie de compra de innovación, es decir, se pretende crear innovación mediante de la reglamentación o compra de equipos, idea poco acertada debido a que la innovación no es un bien tangible y su fomento se da en espacios lúdicos donde equivocarse no es sancionado, como lo que sucede en Silicon Valley en Estados Unidos donde la creación de nuevas tecnologías se realiza a diario por personas que han fallado en la creación de empresas y han resurgido por la perseverancia y el ambiente que reina en el lugar.

Además la problemática se ahonda debido a la importación de unidades funcionales, definición que se aplica cuando una máquina o una combinación de máquinas está constituida por elementos individualizados diseñados para realizar conjuntamente una función específica; esta figura de unidad funcional no permite la desagregación ni la asimilación de tecnología por parte de los países en vías de desarrollo, debido a que, por su naturaleza, permite la importación de varios equipos que dependen uno de otro y pierden la garantía del fabricante si se utiliza un equipo producido localmente.

Adicionalmente, el uso de patentes a nivel mundial pone en riesgo la asimilación de tecnología mediante el método de copiar, igualar y superar; las personas o empresas que quieran desagregar y replicar elementos se exponen a demandas de las grandes firmas que buscan resguardar su inversión guardando la tecnología para fines comerciales y no de avance científico. 
Los análisis de desagregación tecnológica tienen como finalidad la contribución y participación de un Estado en la elaboración de proyectos, esta herramienta debe aportar datos e información que fomente la producción nacional y dinamice el mercado tecnológico de la industria referente a la innovación, la tecnología y procesos, entre otros; sin embargo, no se han establecido directrices, a nivel regional, que permitan estandarizar los procesos de desagregación.

Existen políticas que promueven la innovación y el desarrollo tecnológico en los países de la región sobre la base del Acuerdo de Cartagena, sin embargo la creación de elementos innovadores son exclusivos del sector académico, hay propuestas a nivel local en relación a la creación de centros con equipos destinados a la desagregación tecnológica, los cuales están enfocados a un nivel netamente técnico, sin una adecuada socialización ni participación directa del sector industrial.

Identificar un modelo de desarrollo industrial para el país, junto con estrategias que fomenten la desagregación tecnológica, es la base para el desarrollo de tecnología regional, aplicar este tipo de estrategias provocará la creación de empresas con un alto componente tecnológico que servirán de ancla para desarrollar un ecosistema creativo con productos innovadores. Para plantear este tipo de industria, inicialmente, se requiere de normativa y legislación que mejore el modelo mediante políticas públicas que contemplen una tolerancia al error, permitan la exoneración de impuestos en etapas iníciales de emprendimientos y fomenten el consumo de productos desarrollados en el país.

Actualmente se ha planteado la creación de centros de desagregación tecnológica como impulso de polos industriales y tecnológicos, situación que ha impulsado la producción de paquetes tecnológicos de los sectores priorizados y de compras recurrentes por las entidades públicas locales, que de todas maneras, deben ser repotencializadas, a la par de los programas desarrollados por el gobierno para impulsar la industria nacional.

Vale enfatizar que la descomposición de un objeto de estudio no debe limitarse en el simple despiece de sus partes, sino que debe aportar información necesaria que fomente la producción nacional y dinamice el mercado tecnológico de la industria nacional; la desagregación tecnológica debe ser entendida como una metodología que funcione como herramienta para la re- 
ducción de complejidades en el proceso, así como facilitar la comprensión y definición de cada uno de los componentes de manera ordenada; por lo tanto, es de utilidad para la planificación, coordinación y control en la incorporación de tecnología.

Para que la desagregación sea un éxito, el Estado debe disponer de una capacidad suficiente, lo que significa fortalecer diversas áreas como la educación científica y tecnológica, estudios de administración y gestión, capacidad interna que se relaciona y confluye en un "sistema nacional de tecnología" el cual generaría las pautas para la creación de tecnología acorde a las necesidades de un país.

Según los análisis realizados por el MIPRO y la Universidad de Yachay (Rivera y Zárate 2015), la función de los Centros de Desagregación Tecnológica (CDT) es incrementar la competitividad empresarial local; se demostró que el impacto o nivel de atraso tecnológico afecta directamente al crecimiento económico del país, por lo tanto una mayor productividad tiene una relación directa con la capacidad competitiva de la economía globalizada.

\section{Conclusiones}

Existe legislación que norma la desagregación tecnológica; en tal virtud, decir que esta herramienta es una estrategia para alcanzar la generación de políticas es acertado si el objetivo es la sustitución de importaciones como una profunda transformación de la balanza comercial no petrolera para alcanzar niveles próximos al equilibrio económico y financiero necesarios.

Los desafíos actuales deben orientarse a la conformación de nuevas industrias y la promoción de nuevos sectores con alta productividad, sostenibles y sustentables, con visión territorial que generen encadenamientos, profundizando la inversión mediante la legislación no solo prohibitiva y el impulso a la creación de lugares lúdicos, lo que generaría desde el gobierno central condiciones de competitividad sistémica.

Impulsar la contratación pública con productos nacionales y promover la inversión privada causaría la sustitución de importaciones lo que induciría a la desagregación tecnológica de partes y equipos, la transferencia de tecnología, desarrollo de conocimiento local y la producción nacional diversificada. 


\section{Referencias}

Cadavid, Frank, Olmar Martínez y Xavier Monsalve. 2002. "Modelo de Desagregación para la Transferencia de Tecnologías Limpias en la Universidad Pontificia Bolivariana, Escuela de Formación Avanzada Maestría en Gestión Tecnológica de Medellín”. Biodigital No. 1: 59-81.

Chávez Frías, Hugo e Innovación. 2001. "Ley de Ciencia, Tecnología e Innovación de Venezuela”. CTS+ I: Revista Iberoamericana de Ciencia, Tecnología, Sociedad e Innovación, No. 1: 1-9.

Constitución de la República del Ecuador [2008]. [Quito].

Ecuador. Ley Orgánica del Sistema Nacional de Contratación Pública, publicado en el Registro Oficial, Suplemento (ROS), No. 395 (Quito, 4 de agosto de 2008), art. 6, num. 6: 5-6.

Empresa Pública de Importaciones (EPI). 2014. Desagregación tecnológica en el Ecuador. Quito: SERCOP. Consulta: octubre de 2016. 〈https://importacionresponsable.wordpress. com/2014/03/31/que-es-la-desagregacion-tecnologica/s.

Flores, Fernando. 2008. Desagregación tecnológica. Blogdiario Hispavista. Consulta: noviembre de 2016. 〈http://florflores.blogspot.es/1209563760/desagregacion-tecnologica/〉.

Haugan, Gregory. 2012. Efffective Work Breakdown Structures. Viena: Project Management Essential Library.

Junta del Acuerdo de Cartagena. 1974. Historia documental del Acuerdo de Cartagena. Buenos Aires: BID / INTAL.

Perú, Comisión Permanente del Congreso de la República. 2007. Ley No. 28303: Ley Marco de Ciencia, Tecnología e Innovación Tecnológica. Consulta: enero de 2017. 〈http://www. pcm.gob.pe/wp-content/uploads/2016/06/Ley_28303_Ley_Marco_Ciencia_Tec_Innovacion_Tecnol\%C3\%B3gica.pdf $\%$.

Rivera, Marco, y Ricardo Zárate. 2015. "Desagregación tecnológica de un prototipo de alto impacto desarrollado por el laboratorio de tecnologías de inclusión de la Universidad Politécnica Salesiana”. Tesis de grado. Universidad Politécnica Salesiana, Sede Cuenca. 〈https://dspace.ups.edu.ec/bitstream/123456789/7948/1/UPS-CT004805.pdf〉.

Secretaría Nacional de Planificación y Desarrollo (SENPLADES). 2013. Plan Nacional del Buen Vivir. Quito: SENPLADES. Consulta: septiembre de 2016. «http://www.buenvivir. gob.ec/s. 